\title{
Modeling of Shallow-Water Equations by Using Implicit Higher-Order Compact Scheme with Application to Dam-Break Problem
}

Jafar Bagheri ${ }^{1}$ and Samir K Das ${ }^{2 *}$

${ }^{1}$ Eslamabad-E-Gharab Branch, Islamic Azad University, Islamabad-E-Gharab, Iran

${ }^{2}$ Defence Institute of Advanced Technology, Girinagar, Pune-411025, India

\begin{abstract}
The paper deals with the unsteady two-dimensional (2D) non-linear shallow-water equations (SWE) in conservation-law form to capture the fluid flow in transition. Numerical simulations of dam-break flood wave in channel transitions have been performed for inviscid and incompressible flow by using two new implicit higher-order compact $(\mathrm{HOC})$ schemes. The algorithm is second order accurate in time and fourth order accurate in space, on the nine-point stencil using third order non-centered difference at the wall boundaries. To solve the algebraic system, bi-conjugate gradient stabilized method (BiCGStab) with preconditioning has been employed. Although, both the schemes are able to capture both transient and steady state solution of shallow water equations, the scheme expressed in conservative law form is unconditionally stable. The model results have been validated for dam-break problem and compared with the experimental data for dry and wet bed conditions. The model results are found to be in good agreement with the experimental observations. The proposed scheme is useful to solve to capture flow transition with minimal number of nodal points, particularly for hyperbolic system.
\end{abstract}

Keywords: SWE; HOC; BiCGStab; Dam-break; Conservation-law; Finite difference; Numerical stability

\section{Introduction}

To model the shallow water equations (SWE), various numerical methods such as explicit and implicit finite-difference methods with higher-order compact (HOC) scheme have been used by a number of investigators. The compact scheme is a class of high order finite difference methods, which provides an effective way of joining spectral method for accuracy and robust characteristics of finite difference schemes. The compact scheme uses smaller stencil to approximate leading truncation error terms of the governing equation and to provide better resolution. Such approximation provides a better representation at shorter length scales and is well applicable for the simulation of waves with high frequency. The application of compact scheme for various fluid flow problems can be obtained from the investigations [1-10]. Navon and Riphagen [1] developed compact fourth order algorithm by using alternating-direction implicit finite-difference scheme to solve non-linear shallow-water equations, expressed in conservationlaw form. Abarbanel and Kumar [2] obtained an unconditionally stable HOC scheme for the Euler Equations. Gupta [3] extended the high accuracy approximation method to solve Navier-Stokes equation for lead driven cavity problem. Lele [4] has investigated higher order compact scheme with spectral-like resolution with the approximation of the first and second derivative up to tenth order on a uniform grid. Spotz and Carey [5] developed higher-order difference scheme on compact stencil for boundary value problems and in particular to solve steady stream-function vorticity equations. Li and Tang [6] extended the previous work of Li et al. [11] for steady Navier-Stokes equation to unsteady case by using fourth order accurate compact scheme. Kalita et al. $[7,9]$ proposed a transformation-free HOC for steady convectivediffusion equation and an efficient transient Navier-Stokes solver by using non-uniform grids. Pandit et al. [8] proposed an implicit HOC finite-difference scheme for two-dimensional unsteady Navier-Stokes equations in irregular geometries by using orthogonal grids. Shah et al. [10] developed a time accurate upwind higher order accurate compact finite difference scheme to Navier-Stokes equations by using artificial viscosity approach.
The analysis of dam-break flow is important to capture spatial and temporal evolution of flood event and safety analysis. The dam-break is basically catastrophic failure of dam, leading to uncontrolled release of water causing flood in the downstream region. Several researchers have studied the flood wave caused due to the dam-break either experimentally or numerically. The experimental investigations can be obtained from the literature [12-15]. The successful representation of dam-break flow using numerical simulation have been reported by [16-26]. Townson and Al-Salihi [19] developed dam-break flow model for parallel, converging and diverging boundaries using the method of characteristics. Bellos et al. [20] developed a two-dimensional numerical method for dam-break problem by using the combination of finite-element and finite-difference methods. Mohapatra and Bhallamundi [21] simulated dam-break flow in channel transitions by using MacCormack [27] scheme. Zoppou and Roberts [22] obtained numerical solution for unsteady dam-break flow problem. Arico et al. [23] used shallow water approach for the representation of dambreak flow. The literature on open channel and dam-break flows can be obtained from Chaudhry [24]. Recently, three-dimensional numerical simulations for free surface flows induced by dam-break have been reported by Biscarini et al. [25]. Bellos and Hrissanthou [26] developed two numerical models for one-dimensional Shallow Water Equation (SWE) or Saint-Venants's equation by using Lax-Wendroff [28] and MacCormack [27] schemes.

In the present study, we propose HOC scheme to solve transient

*Corresponding author: Samir K Das, Defence Institute of Advanced Technology, Girinagar, Pune-411025, India, Tel: +91-20-24304081; Fax: +91-20-24389288; E-mail: samirkdas@diat.ac.in / samirkumar_d@yahoo.com

Received May 24, 2013; Accepted June 22, 2013; Published July 07, 2013

Citation: Bagheri J, Das SK (2013) Modeling of Shallow-Water Equations by Using Implicit Higher-Order Compact Scheme with Application to Dam-Break Problem. J Appl Computat Math 2: 132. doi:10.4172/2168-9679.1000132

Copyright: ( 2013 Bagheri J, et al. This is an open-access article distributed unde the terms of the Creative Commons Attribution License, which permits unrestricted use, distribution, and reproduction in any medium, provided the original author and source are credited. 
two-dimensional SWE. The distinct advantage of this scheme is high order accuracy associated with compact stencils which provides accurate numerical solution even for relatively coarser grids. To demonstrate the application of higher order compact scheme, classical dam-break problem is considered to simulate the flood wave in channel transition. We consider parallel-parallel channel with complete breach [21] as shown in Figure 1a, 1b. The flow is considered to be inviscid and incompressible and accordingly the non-linear SWE are expressed as

$$
\begin{aligned}
& \frac{\partial h}{\partial t}+\frac{\partial}{\partial x}(u h)+\frac{\partial}{\partial y}(v h)=0 \\
& \frac{\partial}{\partial t}(u h)+\frac{\partial}{\partial x}\left(u^{2} h\right)+\frac{\partial}{\partial y}(u v h)=g h\left[S_{o_{x}}-\frac{\partial h}{\partial x}-S_{f_{x}}\right] \\
& \frac{\partial}{\partial t}(v h)+\frac{\partial}{\partial x}(u v h)+\frac{\partial}{\partial y}\left(v^{2} h\right)=g h\left[S_{o_{y}}-\frac{\partial h}{\partial y}-S_{f_{y}}\right]
\end{aligned}
$$

These above equations are described in primitive variable form are obtained from Navier-Stokes (NS) equations by integrating over the depth and by assuming hydrostatic pressure distribution. Further, vertical velocity component and corresponding shear stress is also considered insignificant. The set of partial differential equations (PDEs) are nonlinear first-order and hyperbolic in nature. Here, $\mathrm{h}$ is water depth; $\mathrm{u}$ is depth averaged velocity in the $x$-direction; $v$ is depth averaged velocity in the $y$-direction; $g$ is acceleration due to gravity; $S_{o_{x}}$ is bed slope in the $x$-direction; $S_{o_{y}}$ is bed slope in the $y$-direction; $S_{f_{x}}$ is bottom friction in the $x$-direction; $S_{f_{v}}$ is bottom friction in the $y$-direction. Generally, the bottom friction can be estimated by using the Manning's formula:

$$
S_{f_{x}}=\frac{n^{2} u \sqrt{u^{2}+v^{2}}}{c_{0}^{2} h^{1.3 \overline{3}}} \text { and } S_{f_{y}}=\frac{n^{2} v \sqrt{u^{2}+v^{2}}}{c_{0}^{2} h^{1.3 \overline{3}}}
$$

Where $n$ is Manning roughness coefficient; and $c_{0}$ is a dimensional constant. The paper is organized as follows: Section- 2 provides the mathematical formulation and discretization procedure. Section-3 discusses computation procedure and Section- 4 discusses boundary conditions. In Section-5, linearized stability analysis is derived. The numerical results of test cases are given in Section-6. Finally, Section-7 provides results and discussion.

\section{Mathematical Formulation and Discretization Procedure}

The governing equations (1)-(3) can be written in conservative law form or divergence form as

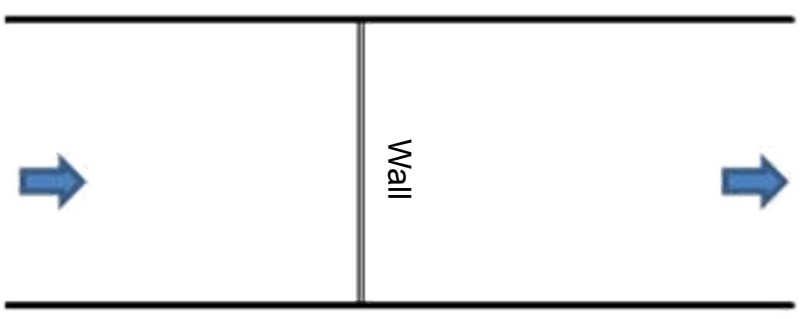

Figure 1: Definition sketch for the transition

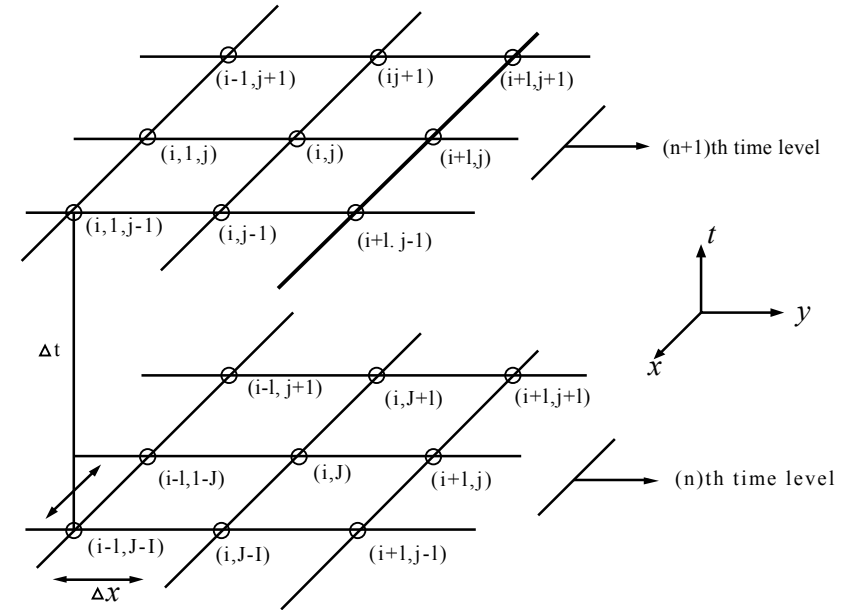

Figure 2: Higher order compact stencil using $(9,9)$ grid points.

$$
\frac{\partial \varphi}{\partial t}+\frac{\partial F(\varphi)}{\partial x}+\frac{\partial G(\varphi)}{\partial y}=S(\varphi)
$$

where $\varphi, F(\varphi), G(\varphi)$ and $S(\varphi)$ are the column matrices

$$
\varphi=\left[\begin{array}{c}
h \\
h u \\
h v
\end{array}\right], F(\varphi)=\left[\begin{array}{c}
u h \\
u^{2} h+g h^{2} / 2 \\
u v h
\end{array}\right], G(\varphi)=\left[\begin{array}{c}
v h \\
u v h \\
v^{2} h+g h^{2} / 2
\end{array}\right], S(\varphi)=\left[\begin{array}{c}
0 \\
g h\left(S_{o_{x}}-S_{f_{x}}\right) \\
g h\left(S_{o_{y}}-S_{f_{y}}\right)
\end{array}\right]
$$

Where $\varphi$ denotes a vector containing the primitive variables $h, u, v$; $\mathrm{F}(\varphi)$ and $\mathrm{G}(\varphi)$ are vectors written in flux form and are functions of $\varphi$; $S(\varphi)$ is the source Term. The scheme developed here is in rectangular Cartesian coordinate system using nine point stencil for grid point $(i, j)$ with two-time levels at $n$ and $n+1$ as shown in Figure 2. We discretize equation (1) by adopting two procedures: (i) non-conservative form and (ii) conservative law form. Both the approaches are applied to solve shallow water equations (SWE). However, conservative law formis used to solve equation (5).

\section{Discretization in non-conservative form}

It is necessary for some numerical schemes to represent the governing equations in non-conservative form. Therefore, after rearranging equation (5) in non-conservation form, one can write

$$
\frac{\partial \varphi}{\partial t}+A \frac{\partial \varphi}{\partial x}+B \frac{\partial \varphi}{\partial y}=S(\varphi)
$$

where A, B are the Jacobian of $\mathrm{F}(\varphi), \mathrm{G}(\varphi)$ with respect to $\varphi$ :

$$
A=\left[\begin{array}{ccc}
0 & 1 & 0 \\
-u^{2}+g h & 2 u & 0 \\
-u v & v & u
\end{array}\right] ; B=\left[\begin{array}{ccc}
0 & 0 & 1 \\
-u v & v & u \\
-v^{2}+g h & 0 & 2 v
\end{array}\right]
$$

First we develop HOC formulation for the steady-state form of equation (7) which is obtained when $\varphi, \mathrm{A}, \mathrm{B}$ and $\mathrm{S}(\varphi)$ are independent of $t$. Accordingly, equation (7) becomes

$$
A \frac{\partial \varphi}{\partial x}+B \frac{\partial \varphi}{\partial y}=S(\varphi)
$$

The rectangular domain is divided in to a uniform mesh size $\Delta x$ and $\Delta y$ along $x$ and $y$ directions respectively; $\varphi_{i j}$ refers to the approximation 
of $\varphi\left(x_{i}, y_{j}\right)$ where $\left(x_{i}, y_{j}\right)$ is the coordinate of a typical node. In the computational domain on a grid point $(i, j)$, the stencil is similar to the one at the $n$ or $(n+1)$ th time level as shown in Figure 2. A second-order central difference approximation is considered by including the leading truncation error terms at the $\left(x_{i}, y_{j}\right)$ th node for the derivatives appears in equation (9)

$$
A_{i j} \delta_{x} \varphi_{i j}+B_{i j} \delta_{y} \varphi_{i j}-\frac{\Delta x^{2}}{6}\left(A \frac{\partial^{3} \varphi}{\partial x^{3}}\right)_{i j}-\frac{\Delta y^{2}}{6}\left(B \frac{\partial^{3} \varphi}{\partial y^{3}}\right)_{i j}=\left.S(\varphi)\right|_{i j}+o\left(\Delta x^{4}, \Delta y^{4}\right)
$$

where $\delta_{x}$ and $\delta_{y}$ are the first order central difference operators along the $x$ and $y$ directions respectively. To obtain a fourth order compact scheme, we approximate the derivatives $\frac{\partial^{3} \varphi}{\partial x^{3}}, \frac{\partial^{3} \varphi}{\partial y^{3}}$ by differentiating equation (9) and include them in the difference equation (10). The successive differentiation of (9) with respect to $x$ yields

$$
\begin{aligned}
& -\left.\left(A \frac{\partial^{3} \varphi}{\partial x^{3}}\right)\right|_{i j}= \\
& \left.\left(\frac{\partial^{2} A}{\partial x^{2}} \frac{\partial \varphi}{\partial x}+2 \frac{\partial A}{\partial x} \frac{\partial^{2} \varphi}{\partial x^{2}}+\frac{\partial^{2} B}{\partial x^{2}} \frac{\partial \varphi}{\partial y}+2 \frac{\partial B}{\partial x} \frac{\partial^{2} \varphi}{\partial y \partial x}+B \frac{\partial^{3} \varphi}{\partial y \partial x^{2}}-\frac{\partial^{2} S(\varphi)}{\partial x^{2}}\right)\right|_{i j} \\
& -\left.\left(B \frac{\partial^{3} \varphi}{\partial y^{3}}\right)\right|_{i j}= \\
& \left.\left(\frac{\partial^{2} B}{\partial y^{2}} \frac{\partial \varphi}{\partial y}+2 \frac{\partial B}{\partial y} \frac{\partial^{2} \varphi}{\partial y^{2}}+\frac{\partial^{2} A}{\partial y^{2}} \frac{\partial \varphi}{\partial x}+2 \frac{\partial A}{\partial y} \frac{\partial^{2} \varphi}{\partial x \partial y}+A \frac{\partial^{3} \varphi}{\partial x \partial y^{2}}-\frac{\partial^{2} S(\varphi)}{\partial y^{2}}\right)\right|_{i j}
\end{aligned}
$$

Using central differencing and writing in operator form, equations (11) and (12) become

$$
\begin{aligned}
& -\left(A \frac{\partial^{3} \varphi}{\partial x^{3}}\right)_{i j}=\left(\left[\begin{array}{l}
\delta_{x}^{2} A \delta_{x}+2 \delta_{x} A \delta_{x}^{2}+\delta_{x}^{2} B \delta_{y}+ \\
2 \delta_{x} B \delta_{x} \delta_{y}+B \delta_{y} \delta_{x}^{2}
\end{array}\right] \varphi_{i j}-\delta_{x}^{2} S_{i j}+O\left(\Delta x^{2}, \Delta y^{2}\right)\right)_{i j} \\
& -\left(B \frac{\partial^{3} \varphi}{\partial y^{3}}\right)_{i j}=\left(\left[\begin{array}{l}
\delta_{y}^{2} B \delta_{y}+2 \delta_{y} B \delta_{y}^{2}+\delta_{y}^{2} A \delta_{x}+ \\
2 \delta_{y} A \delta_{y} \delta_{x}+A \delta_{x} \delta_{y}^{2}
\end{array}\right] \varphi_{i j}-\delta_{y}^{2} S_{i j}+O\left(\Delta x^{2}, \Delta y^{2}\right)\right)_{i j}
\end{aligned}
$$

Substituting equations (13), (14) into equation (10) we obtain the following approximation to (9)

$$
\begin{aligned}
& \mid A_{i j} \delta_{x} \varphi_{i j}+B_{i j} \delta_{y} \varphi_{i j}+\frac{\Delta x^{2}}{6}\left(\left[\begin{array}{l}
\delta_{x}^{2} A \delta_{x}+2 \delta_{x} A \delta_{x}^{2}+\delta_{x}^{2} B \delta_{y}+ \\
2 \delta_{x} B \delta_{x} \delta_{y}+B \delta_{y} \delta_{x}^{2}
\end{array}\right] \varphi_{i j}-\delta_{x}^{2} S_{i j}+O\left(\Delta x^{2}, \Delta y^{2}\right)_{i j}\right)_{i j} \\
& +\frac{\Delta y^{2}}{6}\left(\left[\delta_{y}^{2} B \delta_{y}+2 \delta_{y} B \delta_{y}^{2}+\delta_{y}^{2} A \delta_{x}+2 \delta_{y} A \delta_{y} \delta_{x}+A \delta_{x} \delta_{y}^{2}\right] \varphi_{i j}-\delta_{y}^{2} S_{i j}+O\left(\Delta x^{2}, \Delta y^{2}\right)\right)_{i j} \\
& =\left.S(\varphi)\right|_{i j}+O\left(\Delta x^{4}, \Delta y^{4}\right)_{i j}
\end{aligned}
$$

After rearranging, we obtain

$$
\begin{aligned}
& C_{i j} \delta_{x} \varphi_{i j}+D_{i j} \delta_{y} \varphi_{i j}+F_{i j} \delta_{x}^{2} \varphi_{i j}+G_{i j} \delta_{y}^{2} \varphi_{i j}+\frac{1}{6} \\
& {\left[\Delta y^{2} A_{i j} \delta_{x} \delta_{y}^{2}+\Delta x^{2} B_{i j} \delta_{y} \delta_{x}^{2}+E_{i j} \delta_{x} \delta_{y}\right] \varphi_{i j}=\overline{S_{i j}}+O\left(\Delta x^{4}, \Delta y^{4}\right)_{i j}}
\end{aligned}
$$

where the coefficients $A_{i j}, B_{i j}, C_{i j}, D_{i j}, E_{i j}, F_{i j}, G_{i j}$ and $\bar{S}_{i j}$ are as follows:

$$
\begin{aligned}
& C_{i j}=A_{i j}+\frac{\Delta x^{2}}{6} \delta_{x}^{2} A_{i j}+\frac{\Delta y^{2}}{6} \delta_{y}^{2} A_{i j} \\
& D_{i j}=B_{i j}+\frac{\Delta x^{2}}{6} \delta_{x}^{2} B_{i j}+\frac{\Delta y^{2}}{6} \delta_{y}^{2} B_{i j} \\
& E_{i j}=2 \Delta x^{2} \delta_{x} B_{i j}+2 \Delta y^{2} \delta_{y} A_{i j}
\end{aligned}
$$

$$
\begin{aligned}
& F_{i j}=\frac{\Delta x^{2}}{3} \delta_{x} A_{i j} \\
& G_{i j}=\frac{\Delta y^{2}}{3} \delta_{y} B_{i j} \\
& \bar{S}_{i j}=S_{i j}+\frac{\Delta x^{2}}{6} \delta_{x}^{2} S_{i j}+\frac{\Delta y^{2}}{6} \delta_{y}^{2} S_{i j}
\end{aligned}
$$

To derive a finite difference scheme for the unsteady case, we replace the source function $S(\varphi)$ in equation (9) by $S(\varphi)-\frac{\partial \varphi}{\partial t}$ and approximate the time derivative term with forward difference whence equation (16) yields

$$
\begin{aligned}
& C_{i j} \delta_{x} \varphi_{i j}+D_{i j} \delta_{y} \varphi_{i j}+F_{i j} \delta_{x}^{2} \varphi_{i j}+G_{i j} \delta_{y}^{2} \varphi_{i j}+\frac{1}{6}\left[\Delta y^{2} A_{i j} \delta_{x} \delta_{y}^{2}+\Delta x^{2} B_{i j} \delta_{y} \delta_{x}^{2}+E_{i j} \delta_{x} \delta_{y}\right] \varphi_{i j}= \\
& \left(S_{i j}-\left.\frac{\partial \varphi}{\partial t}\right|_{i j}\right)+\frac{\Delta x^{2}}{6} \delta_{x}^{2}\left(S_{i j}-\left.\frac{\partial \varphi}{\partial t}\right|_{i j}\right)+\frac{\Delta y^{2}}{6} \delta_{y}^{2}\left(S_{i j}-\left.\frac{\partial \varphi}{\partial t}\right|_{i j}\right)+O\left(\Delta x^{4}, \Delta y^{4}\right)
\end{aligned}
$$

After rearranging, equation (23) can be finally written as

$$
\begin{aligned}
& {\left[1+\frac{\Delta x^{2}}{6} \delta_{x}^{2}+\frac{\Delta y^{2}}{6} \delta_{y}^{2}\right] I \delta_{t}^{+} \varphi_{i j}^{n}+C_{i j} \delta_{x} \varphi_{i j}^{n}+D_{i j} \delta_{y} \varphi_{i j}^{n}+F_{i j} \delta_{x}^{2} \varphi_{i j}^{n}+G_{i j} \delta_{y}^{2} \varphi_{i j}^{n}} \\
& +\frac{1}{6}\left[\Delta y^{2} A_{i j} \delta_{x} \delta_{y}^{2}+\Delta x^{2} B_{i j} \delta_{y} \delta_{x}^{2}+E_{i j} \delta_{x} \delta_{y}\right] \varphi_{i j}^{n}=\bar{S}_{i j}+O\left(\Delta x^{4}, \Delta y^{4}\right)
\end{aligned}
$$

where

$$
\delta_{t}^{+} \varphi_{i j}^{n}=\frac{\varphi_{i j}^{n+1}-\varphi_{i j}^{n}}{\Delta t}
$$

and $\mathrm{I}$ is the identity matrix, $n$ represents the time level and the coefficients $\mathrm{A}_{\mathrm{ij}}, \mathrm{B}_{\mathrm{ij}}, \mathrm{C}_{\mathrm{ij}}, \mathrm{D}_{\mathrm{ij}}, \mathrm{E}_{\mathrm{ij}}, \mathrm{F}_{\mathrm{ij}}, \mathrm{G}_{\mathrm{ij}}$ and $\overline{\mathrm{S}}_{\mathrm{ij}}$ to be evaluated at the time level $n$. We introduce a weighted average parameter $\mu$ for the approximation of time derivative with differencing $t_{\mu}=(1-\mu) t^{n}+\mu t^{n+1}$, where $0 \leq \mu$ $\leq 1$. Varying $\mu$ leads to different schemes of different time accuracies. Accordingly, equation (24) becomes

$$
\begin{aligned}
& {\left[1+\frac{\Delta x^{2}}{6} \delta_{x}^{2}+\frac{\Delta y^{2}}{6} \delta_{y}^{2}\right] I \delta_{t}^{+} \varphi_{i j}^{n}+} \\
& (1-\mu)\left[\begin{array}{l}
C_{i j} \delta_{x} \varphi_{i j}^{n}+D_{i j} \delta_{y} \varphi_{i j}^{n}+F_{i j} \delta_{x}^{2} \varphi_{i j}^{n}+G_{i j} \delta_{y}^{2} \varphi_{i j}^{n}+\frac{1}{6} \\
{\left[\Delta y^{2} A_{i j} \delta_{x} \delta_{y}^{2}+\Delta x^{2} B_{i j} \delta_{y} \delta_{x}^{2}+E_{i j} \delta_{x} \delta_{y}\right] \varphi_{i j}^{n}}
\end{array}\right] \\
& +\mu\left[\begin{array}{l}
C_{i j} \delta_{x} \varphi_{i j}^{n+1}+D_{i j} \delta_{y} \varphi_{i j}^{n+1}+F_{i j} \delta_{x}^{2} \varphi_{i j}^{n+1}+G_{i j} \delta_{y}^{2} \varphi_{i j}^{n+1}+\frac{1}{6} \\
{\left[\Delta y^{2} A_{i j} \delta_{x} \delta_{y}^{2}+\Delta x^{2} B_{i j} \delta_{y} \delta_{x}^{2}+E_{i j} \delta_{x} \delta_{y}\right] \varphi_{i j}^{n+1}}
\end{array}\right] \\
& =(1-\mu) \bar{S}_{i j}^{n}+\mu \bar{S}_{i j}^{n+1}+O\left((\Delta t)^{p}, \Delta x^{4}, \Delta y^{4}\right)
\end{aligned}
$$

This provides a class of integrators, i.e., for $\mu=0, p=1$ gives forward Euler, $\mu=1, p=1$ gives backward Euler and $\mu \mu=1 / 2, p=2$ gives CrankNicolson. Equation (26) can be written as

$$
\begin{aligned}
& \sum_{k_{1}-1}^{1} \sum_{k_{2}-1}^{1} w_{i+k_{1} j+k_{2}} \varphi_{i+k_{1} j+k_{2}}^{(n+1)}= \\
& \sum_{k_{1}-1}^{1} \sum_{k_{2}-1}^{1} w_{i+k_{1} j+k_{2}} \varphi_{i+k_{1}}^{(n)} j+k_{2} \\
& +24 \Delta t\left(\mu \bar{S}_{i j}^{(n+1)}+24(1-\mu) \bar{S}_{i j}^{(n)}+O\left((\Delta t)^{p}, h^{4}, k^{4}\right)\right)
\end{aligned}
$$

where 


$$
w_{i+k_{1} j+k_{2}}=\mu \frac{\Delta t}{\Delta x^{2} \Delta y^{2}} p_{i+k_{1} j+k_{2}}+q_{i+k_{1} j+k_{2}}
$$

and

$$
\begin{aligned}
& w_{i+k_{1} j+k_{2}}^{\prime}=(\mu-1) \frac{\Delta t}{\Delta x^{2} \Delta y^{2}} p_{i+k_{1} j+k_{2}}+q_{i+k_{1} j+k_{2}} \\
& p_{i-1 j-1}=-2 X Y 1_{i j}+2 E 1_{i j} q_{i-1 j-1}=0 \\
& p_{i j-1}=-2 Y Y 1_{i j}+2 G 1_{i j} q_{i j-1}=4 I \\
& p_{i+1 j-1}=-2 X Y 2_{i j}-2 E 1_{i j} q_{i+1 j-1}=0 \\
& p_{i-1 j}=-2 X X 1_{i j}+2 F 1_{i j} q_{i-1 j}=4 I \\
& p_{i j}=-F 1_{i j}-G 1_{i j} q_{i j}=8 I \\
& p_{i+1 j}=2 X X 1_{i j}+2 F 1_{i j} q_{i+1 j}=4 I \\
& p_{i-1 j+1}=-2 X Y 2_{i j}-2 E 1_{i j} q_{i-1 j+1}=0 \\
& p_{i j+1}=2 Y Y 1_{i j}+2 G 1_{i j} q_{i j+1}=4 I \\
& p_{i+1 j+1}=2 X Y 1_{i j}+2 E 1_{i j} q_{i+1 j+1}=0
\end{aligned}
$$

where

$$
\begin{aligned}
& X Y 1_{i j}=\Delta x \Delta y^{2} A_{i j}+\Delta x^{2} \Delta y B_{i j} \\
& X Y 2_{i j}=\Delta x \Delta y^{2} A_{i j}-\Delta x^{2} \Delta y B_{i j} \\
& Y Y 1_{i j}=6 \Delta x^{2} \Delta y D_{i j}-2 \Delta x^{2} \Delta y B_{i j} \\
& X X 1_{i j}=6 \Delta x \Delta y^{2} C_{i j}-2 \Delta x \Delta y^{2} A_{i j} \\
& E 1_{i j}=3 \Delta x \Delta y E_{i j} \\
& G 1_{i j}=12 \Delta x^{2} G_{i j} \\
& F 1_{i j}=12 \Delta y^{2} F_{i j}
\end{aligned}
$$

Equation (27) represents HOC finite difference approximation for the two-dimensional unsteady SWE. For uniform grids, the spatial accuracy of the proposed scheme becomes fourth order accurate.

\section{Discretization in conservation form}

From equation (5) the steady-state form becomes

$$
\frac{\partial F(\varphi)}{\partial x}+\frac{\partial G(\varphi)}{\partial y}=S(\varphi)
$$

We consider a second-order central difference approximation by including the leading truncation error terms at the $\left(x_{i}, y_{j}\right)$ the node for the derivatives appears in (32)

$$
\delta_{x} F_{i j}(\varphi)-\frac{\Delta x^{2}}{6}\left(\frac{\partial^{3} F_{i j}(\varphi)}{\partial x^{3}}\right)_{i j}-\frac{\Delta y^{2}}{6}\left(\frac{\partial^{3} G_{i j}(\varphi)}{\partial y^{3}}\right)_{i j}=\left.S(\varphi)\right|_{i j}+O\left(\Delta x^{4}, \Delta y^{4}\right)
$$

The successive differentiation of (32) with respect to $\mathrm{x}$ and $\mathrm{y}$ yields

$$
\frac{\partial^{3} F(\varphi)}{\partial x^{3}}=\frac{\partial^{2} S}{\partial x^{2}}-\frac{\partial^{3} G(\varphi)}{\partial y \partial x^{2}} ; \frac{\partial^{3} G(\varphi)}{\partial y^{3}}=\frac{\partial^{2} S}{\partial y^{2}}-\frac{\partial^{3} F(\varphi)}{\partial x \partial y^{2}}
$$

By substituting equation (34) in (33) we get

$$
A_{i j} \delta_{x} \varphi_{i j}+B_{i j} \delta_{y} \varphi_{i j}=\bar{S}_{i j}+O\left(\Delta x^{4}, \Delta y^{4}\right)
$$

For unsteady case, we replace the source function $S(\varphi)$ by $S(\varphi)-\frac{\partial \varphi}{\partial t}$ and approximate the time derivative term with forward difference. Using similar approach, we notice that the equations (27), (28), (29) and (30) remain unchanged whereas equations (31) change along with the matrices $C_{i j}, D_{i j}, E_{i j}, F_{i j}, G_{i j}$ and these are

$$
\begin{aligned}
& C_{i j}=A_{i j}, D_{i j}=B_{i j}, E_{i j}=0, F_{i j}=0, G_{i j}=0 \\
& \bar{S}_{i j}=S_{i j}+\frac{\Delta x^{2}}{6} \delta_{x}^{2} S_{i j}+\frac{\Delta y^{2}}{6} \delta_{y}^{2} S_{i j}+\bar{Z} \\
& \bar{Z}=\frac{\Delta y^{2}}{6} \delta_{x} \delta_{y}^{2} F(\varphi)+\frac{\Delta x^{2}}{6} \delta_{y} \delta_{x}^{2} G(\varphi) \\
& X Y 1_{i j}=0 \\
& X Y 2_{i j}=0 \\
& Y Y 1_{i j}=6 \Delta x^{2} \Delta y B_{i j} \\
& X X 1_{i j}=6 \Delta x \Delta y^{2} A_{i j} \\
& E 1_{i j}=0 \\
& G 1_{i j}=0 \\
& F 1_{i j}=0
\end{aligned}
$$

\section{Computational Procedures and Algorithm}

To solve the algebraic systems of equation (27), the following computational procedures are adopted. Using the proposed finite difference approximation, the system of equation (27) can be written in matrix form as

$$
M \sigma^{n+1}=f\left(\sigma^{n}\right)
$$

Where $\mathrm{M}$ is a coefficient matrix. For the coefficient matrix $\mathrm{M}$ with grid size of $(m \times n)$, the structure is 9-band block matrix whose elements are also $3 \times 3$ matrices with $(3 \times m \times n)$ dimension. $\sigma^{\mathrm{n}+1}$ and $f\left(\sigma^{\mathrm{n}}\right)$ are $(3 \times m \times n)$ component vectors can be expressed as

$$
\sigma=\left(\varphi_{11}, \varphi_{12}, \ldots . ., \varphi_{1 n}, \varphi_{21}, \varphi_{22}, \ldots ., \varphi_{2 n}, \ldots ., \varphi_{m 1}, \varphi_{m 1}, \ldots . ., \varphi_{m n}\right)
$$

Where $\varphi_{i j}$ are three-element vectors of flow variables $h, h u, h v$. It can be mentioned here that after each iteration, the coefficient matrix $M$ changes. We use bi-conjugate gradient stabilized method (BiCGStab) with preconditioning. It is also possible to partition the coefficient matrix $\mathrm{M}$ to a block tridiagonal matrix with its elements $(3 \times n) \times(3 \times n)$ matrix where $m \times n$ is the grid size. Equation (42) shows the block tridiagonal matrix for grid size $5 \times 5$ as an example with its elements $15 \times 15$ with Neumann boundary conditions at the side walls and Dirichlet boundary conditions at the inflow and outflow boundaries.

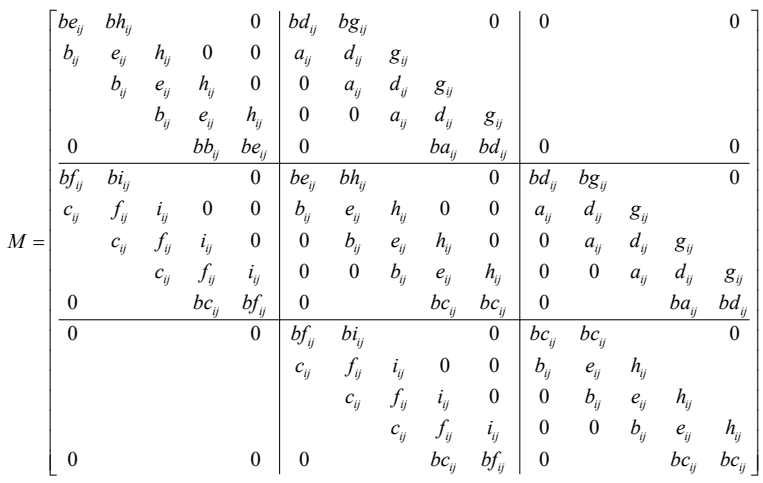

\section{Solution algorithm}

Step 1: Initialize the height and velocity fields 
Step 2: Evaluate the variable coefficient matrix $M$ and variable coefficient $f\left(\sigma^{\mathrm{n}}\right)$ in (27) using equation (40) and boundary conditions (52) and (53). The source term in $n$ and $n+1$ time level is evaluated with one step delay. $\left(\sigma^{\mathrm{n}}\right)$.

Step 3: Using BiCGStab algorithm to solve system (40), $M \sigma^{\mathrm{n}+1}=f$

Step 4: Filter out short-wave noise from the $\mathrm{u}, \mathrm{v}$, and $\mathrm{h}$ using Wallington filter (70)

Step 5: Store $\sigma$ and time step in a file.

Step 6: Increase time step by $\Delta t$, if $\mathrm{t} \leq \mathrm{t}_{\max }$ go to Step 2 .

Step 7: End.

\section{Compact Boundary Conditions}

To solve discretized equation (27), Dirichlet boundary conditions for inflow and outflow and Neumann boundary conditions for both the walls are considered. Prior to dam breach the upstream section acts as a reservoir. Therefore, Dirichlet boundary condition is applied at the downstream end after the dam breach. Neumann boundary conditions are applied in reflective or solid boundaries. The advantage of Dirichlet conditions is that they are simple and straightforward and relatively easy to implement in a HOC setting. We implement Adam [29] boundary conditions and Taylor series analysis of backward and forward difference to develop HOC wall boundary conditions for $\partial \varphi / \partial y$. However, for Neumann type conditions, it is possible to use one order lower in accuracy at the boundary approximations although such approximations to the interior points remain unaffected [30]. We adopt two boundary conditions Adam [31] and Taylor series analysis for HOC approximations for wall boundaries.

\section{Adam boundary condition}

Using the Adam boundary conditions [29], HOC approximation at the walls and interior points in $\mathrm{y}$-direction become

$$
\begin{aligned}
& \left(\frac{\partial \varphi}{\partial y}\right)_{i 1}+2\left(\frac{\partial \varphi}{\partial y}\right)_{i 2}=\frac{1}{2 \Delta y}\left(-5 \varphi_{i 1}+4 \varphi_{i 2}+\varphi_{i 3}\right)+O\left(\Delta y^{3}\right) \\
& \left(\frac{\partial \varphi}{\partial y}\right)_{i 3}+2\left(\frac{\partial \varphi}{\partial y}\right)_{i 2}=\frac{1}{2 \Delta y}\left(-\varphi_{i 1}-4 \varphi_{i 2}+5 \varphi_{i 3}\right)+O\left(\Delta y^{3}\right) \\
& \left(\frac{\partial \varphi}{\partial y}\right)_{i n}+2\left(\frac{\partial \varphi}{\partial y}\right)_{i n-1}=\frac{1}{2 \Delta y}\left(5 \varphi_{i n}-4 \varphi_{i n-1}-\varphi_{i n-2}\right)+O\left(\Delta y^{3}\right) \\
& \left(\frac{\partial \varphi}{\partial y}\right)_{i n-2}+2\left(\frac{\partial \varphi}{\partial y}\right)_{i n-1}=\frac{1}{2 \Delta y}\left(\varphi_{i n}+4 \varphi_{i n-1}-5 \varphi_{i n-2}\right)+O\left(\Delta y^{3}\right)
\end{aligned}
$$

Here $j=1$ and $j=\mathrm{n}$ indicate the left and right side walls respectively.

Using equation (7), we can write

$$
B \frac{\partial \varphi}{\partial y}=S(\varphi)-\frac{\partial \varphi}{\partial t}-A \frac{\partial \varphi}{\partial x}
$$

By applying the solid wall and symmetry boundaries and using equation (43), we obtain

$$
0+2\left(\frac{\partial \varphi}{\partial y}\right)_{i 2}=\frac{1}{2 \Delta y}\left(-5 \varphi_{i 1}+4 \varphi_{i 2}+\varphi_{i 3}\right)+O\left(\Delta y^{3}\right)
$$

Substituting equation (44), (45) into (43), gives

$$
\left(S(\varphi)-\frac{\partial \varphi}{\partial t}-A \frac{\partial \varphi}{\partial x}\right)_{i 3}=\frac{1}{2 \Delta y} B_{i 3}\left(4 \varphi_{i 1}-8 \varphi_{i 2}+4 \varphi_{i 3}\right)+O\left(\Delta y^{3}\right)
$$

$$
-\varphi_{i 3}^{n+1}=\left(\frac{\Delta t}{2 \Delta y} B_{i 3}\left(4 \varphi_{i 1}-8 \varphi_{i 2}+4 \varphi_{i 3}\right)\right)^{n}-\Delta t S_{i 3}+\Delta t A_{i 3}\left(\frac{\varphi_{i+13}-\varphi_{i-13}}{2 \Delta x}\right)-\varphi_{i 3}^{n}+O\left(\Delta y^{3}\right)
$$

Similarly

$$
\begin{aligned}
& -\varphi_{i n-2}^{n+1}=\left(\frac{\Delta t}{2 \Delta y} B_{i n-2}\left(-4 \varphi_{i n}+8 \varphi_{i n-1}-4 \varphi_{i n-2}\right)\right)^{n}-\Delta t S_{i n-2} \\
& +\Delta t A_{i n-2}\left(\frac{\varphi_{i+1 n-2}-\varphi_{i-1 n-2}}{2 \Delta x}\right)-\varphi_{i n-2}^{n}+O\left(\Delta y^{3}\right)
\end{aligned}
$$

\section{Taylor series analysis}

Using Taylor's series analysis and using the forward difference for $j=1$, we get

$$
\left(\frac{\partial \varphi}{\partial y}\right)_{i 1}=0=\left(\delta^{+} \varphi-\frac{\Delta y}{2} \frac{\partial^{2} \varphi}{\partial y^{2}}-\frac{\Delta y^{2}}{6} \frac{\partial^{3} \varphi}{\partial y^{3}}\right)_{i 1}+O\left(\Delta y^{3}\right)
$$

Using similar approach as described in equation (27) with $\mu=0$, we get

$$
\begin{aligned}
& B_{i 1} \frac{\varphi_{i 2}-\varphi_{i 1}}{\Delta y}+\left(-\frac{\Delta y}{2}\left(\frac{\partial S}{\partial y}-\frac{\partial A}{\partial y} \frac{\partial \varphi}{\partial x}-A \frac{\partial^{2} \varphi}{\partial y \partial x}\right)-\left.\frac{\Delta y^{2}}{6}\left(\begin{array}{c}
\left.\frac{\partial^{2} S}{\partial y^{2}}-2 \frac{\partial B}{\partial y} \frac{\partial^{2} \varphi}{\partial y^{2}}-\frac{\partial^{2} A}{\partial y^{2}} \frac{\partial \varphi}{\partial x}-\right)\left.\right|_{A \frac{\partial^{3} \varphi}{\partial y^{2} \partial x}-2 \frac{\partial A}{\partial y} \frac{\partial^{2} \varphi}{\partial x \partial y}}
\end{array}\right)\right|_{i 1}=0\right. \\
& \frac{\Delta y}{2} \delta_{y} \delta_{t} \varphi_{i 1}+\frac{\Delta y^{2}}{6} \delta_{y}^{2} \delta_{t} \varphi_{i 1}+B_{i 1} \frac{\varphi_{i 2}-\varphi_{i 1}}{\Delta y}+ \\
& \left.\left(\left(\frac{\Delta y}{2} \frac{\partial A}{\partial y}+\frac{\Delta y^{2}}{6} \frac{\partial^{2} A}{\partial y^{2}}\right) \frac{\partial \varphi}{\partial x}+2 \frac{\Delta y^{2}}{6} \frac{\partial B}{\partial y} \frac{\partial^{2} \varphi}{\partial y^{2}}+\frac{\Delta y^{2}}{6} A \frac{\partial^{3} \varphi}{\partial y^{2} \partial x}+\left(\frac{\Delta y}{2} A+\frac{\Delta y^{2}}{3} \frac{\partial A}{\partial y}\right) \frac{\partial^{2} \varphi}{\partial y \partial x}\right)\right|_{i 1} \\
& =\left.\left(\frac{\Delta y}{2} \frac{\partial S}{\partial y}+\frac{\Delta y^{2}}{6} \frac{\partial^{2} S}{\partial y^{2}}\right)\right|_{i 1} \\
& \left(\frac{I}{6 \Delta t}\right) \varphi_{i 2}^{n+1}+\left(-\frac{I}{3 \Delta t}\right) \varphi_{i 1}^{n+1}+\left(\frac{I}{6 \Delta t}\right) \varphi_{i 3}^{n+1}=\left(\left(\frac{1}{6 \Delta x}+\frac{\Delta y}{2}\right) A+\frac{\Delta y^{2}}{3} \frac{\partial A}{\partial y}\right)_{i 1} \varphi_{i+1,2}^{n}+ \\
& \left(\left(-\frac{1}{6 \Delta x}-\frac{\Delta y}{2}\right) A-\frac{\Delta y^{2}}{3} \frac{\partial A}{\partial y}\right)_{i 1} \varphi_{i-1,2}^{n}+\left(\left(\frac{\Delta y}{2}+\frac{\Delta y^{2}}{3}\right) \frac{\partial A}{\partial y}+\frac{\Delta y^{2}}{6} \frac{\partial^{2} A}{\partial y^{2}}+\left(-\frac{1}{12 \Delta x}-\frac{\Delta y}{2}\right) A\right)_{i 1} \varphi_{i+1,1}^{n} \\
& -\left(\left(\frac{\Delta y}{2}+\frac{\Delta y^{2}}{3}\right) \frac{\partial A}{\partial y}+\frac{\Delta y^{2}}{6} \frac{\partial^{2} A}{\partial y^{2}}+\left(-\frac{1}{12 \Delta x}+\frac{\Delta y}{2}\right) A\right)_{i 1} \varphi_{i-1,1}^{n}+\left(\frac{I}{6 \Delta t}-\frac{B}{\Delta y}+\frac{2}{3} \frac{\partial B}{\partial y}\right)_{i 1} \varphi_{i 2}^{n} \\
& +\left(\frac{1}{12 \Delta x} A\right)_{i 1} \varphi_{i-1,3}^{n}+\left(-\frac{1}{12 \Delta x} A\right)_{i 1} \varphi_{i+1,3}^{n}+\left(-\frac{I}{3 \Delta t}+\frac{B}{\Delta y}-\frac{1}{3} \frac{\partial B}{\partial y}\right)_{i 1} \varphi_{i 1}^{n}+\left(\frac{I}{6 \Delta t}-\frac{1}{3} \frac{\partial B}{\partial y}\right)_{i 1} \varphi_{i 3}^{n} \\
& +\left(\frac{\Delta y}{2} \frac{\partial S}{\partial y}+\frac{\Delta y^{2}}{6} \frac{\partial^{2} S}{\partial y^{2}}\right)_{i 1}
\end{aligned}
$$

Similarly at $j=n$

$$
\begin{aligned}
& \left(\frac{I}{6 \Delta t}\right) \varphi_{i n-1}^{n+1}+\left(-\frac{I}{3 \Delta t}\right) \varphi_{i n}^{n+1}+\left(\frac{I}{6 \Delta t}\right) \varphi_{i n-2}^{n+1}=\left(\left(\frac{1}{6 \Delta x}+\frac{\Delta y}{2}\right) A+\frac{\Delta y^{2}}{3} \frac{\partial A}{\partial y}\right)_{i n} \varphi_{i t, n-1}^{n}+ \\
& \left(\left(-\frac{1}{6 \Delta x}-\frac{\Delta y}{2}\right) A-\frac{\Delta y^{2}}{3} \frac{\partial A}{\partial y}\right)_{i n} \varphi_{i-1, n-1}^{n}+\left(\left(-\frac{\Delta y}{2}+\frac{\Delta y^{2}}{3}\right) \frac{\partial A}{\partial y}+\frac{\Delta y^{2}}{6} \frac{\partial^{2} A}{\partial y^{2}}+\left(-\frac{1}{12 \Delta x}-\frac{\Delta y}{2}\right) A\right)_{i n} \varphi_{i+1, n}^{n} \\
& +\left(\left(-\frac{\Delta y}{2}+\frac{\Delta y^{2}}{3} \frac{\partial A}{\partial y}\right) \frac{\partial A}{\partial y}+\frac{\Delta y^{2}}{6} \frac{\partial^{2} A}{\partial y^{2}}\left(-\frac{1}{12 \Delta x}-\frac{\Delta y}{2}\right) A\right)_{i n} \varphi_{i-1, n}^{n}+\left(\frac{I}{6 \Delta t}+\frac{B}{\Delta y}+\frac{2}{3} \frac{\partial B}{\partial y}\right)_{i n} \varphi_{i n-1}^{n} \\
& +\left(\frac{1}{12 \Delta x} A\right)_{i n} \varphi_{i-1, n-2}^{n}+\left(-\frac{1}{12 \Delta x} A\right)_{i n} \varphi_{i+1, n-2}^{n}+\left(-\frac{I}{3 \Delta t}-\frac{B}{\Delta y}-\frac{1}{3} \frac{\partial B}{\partial y}\right)_{i n} \varphi_{i n}^{n}+\left(\frac{I}{6 \Delta t}-\frac{1}{3} \frac{\partial B}{\partial y}\right)_{i n} \varphi_{i n-2}^{n} \\
& =\left(-\frac{\Delta y}{2} \frac{\partial S}{\partial y}+\frac{\Delta y^{2}}{6} \frac{\partial^{2} S}{\partial y^{2}}\right)_{i n}
\end{aligned}
$$

The following linear extrapolation equations are used at both the boundaries

$$
\varphi_{i 3}^{n}=2 \varphi_{i 2}^{n}-\varphi_{i 1}^{n}, \varphi_{i n-2}^{n}=2 \varphi_{i n-1}^{n}-\varphi_{i n}^{n}
$$

\section{Stability Analysis}

We carry out a von Neuman analysis in the usual manner by casting 
Citation: Bagheri J, Das SK (2013) Modeling of Shallow-Water Equations by Using Implicit Higher-Order Compact Scheme with Application to Dam-Break Problem. J Appl Computat Math 2: 132. doi:10.4172/2168-9679.1000132

Page 6 of 9

with setting $S=0$ in Fourier space. A typical Fourier component of

$$
\varphi_{i j}^{n+1} \text { is given by } \varphi_{i j}^{n+1}=\tilde{\varphi}^{n+1} e^{i j \theta} e^{i k \gamma}
$$

Where $i=\sqrt{-1}, \theta=p \Delta y, \gamma=q \Delta y ; p, q$ are the wave numbers in $x$ and $y$ direction respectively. Substituting equation (55) in (27), we obtain

$$
\begin{aligned}
& {\left[\begin{array}{l}
(8+8 \cos \theta+8 \cos \gamma) I+\mu \frac{\Delta t}{\Delta x^{2} \Delta y^{2}} \\
\left(4 i \sin (\theta+\gamma) X Y 1_{i j}+4 \cos (\theta+\gamma) E 1_{i j}+4 i \sin (\theta-\gamma) X Y 2_{i j}-\right.
\end{array}\right.} \\
& \left.\left.\left.4 \cos (\theta-\gamma) E 1_{i j}+4 i \sin \gamma Y Y 1_{i j}+4 \cos \gamma G 1_{i j}+4 i \sin \theta X X 1_{i j}+4 \cos \theta F 1_{i j}-F 1_{i j}-G 1_{i j}\right)\right]\right)^{n+1} \\
& =\left[(8+8 \cos \theta+8 \cos \gamma) I+(\mu-1) \frac{\Delta t}{\Delta x^{2} \Delta y^{2}}\right. \\
& \left(4 i \sin (\theta+\gamma) X Y 1_{i j}+4 \cos (\theta+\gamma) E 1_{i j}+4 i \sin (\theta-\gamma) X Y 2_{i j}-\right. \\
& \left.\left.4 \cos (\theta-\gamma) E 1_{i j}+4 i \sin \gamma Y Y 1_{i j}+4 \cos \gamma G 1_{i j}+4 i \sin \theta X X 1_{i j}+4 \cos \theta F 1_{i j}-F 1_{i j}-G 1_{i j}\right)\right] \tilde{\varphi}^{n}
\end{aligned}
$$

And the amplification matrix $\widetilde{G}$ is given by

$\widetilde{G}=\tilde{\varphi}^{n+1} / \tilde{\varphi}^{n}=(I-(1-\mu) T) /(I+\mu T)$

Where

$$
\begin{aligned}
& T_{i j}=\frac{\Delta t}{8 \Delta x^{2} \Delta y^{2}(1+\cos \theta+\cos \gamma)} \\
& {\left[4 i \sin (\theta+\gamma) X Y 1_{i j}+4 \cos (\theta+\gamma) E 1_{i j}+4 i \sin (\theta-\gamma) X Y 2_{i j}-\right.} \\
& 4 \cos (\theta-\gamma) E 1_{i j}+4 i \sin \gamma Y Y 1_{i j}+4 \cos \gamma G 1_{i j} \\
& \left.+4 i \sin \theta X X 1_{i j}+4 \cos \theta F 1_{i j}-F 1_{i j}-G 1_{i j}\right]
\end{aligned}
$$

After simplification, we obtain

$$
\begin{aligned}
& T_{i j}=\frac{\Delta t}{\Delta x^{2} \Delta y^{2}(8+8 \cos \theta+8 \cos \gamma)} \\
& {\left[4 i \sin \theta \cos \gamma\left(X Y 1_{i j}+X Y 2_{i j}\right)+4 i \sin \gamma \cos \theta\left(X Y 1_{i j}-X Y 2_{i j}\right)+\right.}
\end{aligned}
$$

$\left.4 i \sin \gamma Y Y 1_{i j}+4 i \sin \theta X X 1_{i j}+4 \cos \gamma G 1_{i j}+8 \cos \theta \cos \gamma E 1_{i j}+4 \cos \theta F 1_{i j}-F 1_{i j}-G 1_{i j}\right]$

For stability, all the eigenvalues of amplitude matrix $\widetilde{G}$ must be on the unit circle. If $\mathrm{ti}, \mathrm{i}=1,2,3$ are the eigenvalues of $\mathrm{T}$ and $\widetilde{g}_{i}, i=1,2,3$ are the eigenvalues of $\widetilde{G}$ then $\tilde{g}_{i}$ can be written as Henrici [31]

$$
\tilde{g}_{i}=\frac{1-(1-\mu) t_{i}}{1+\mu t_{i}}
$$

We consider two cases corresponding to non-conservative and conservative forms.

\section{Analysis for non-conservative form}

In this case, the eigen values of matrix $\mathrm{T}$ can be defined as real and imaginary parts as

$$
t_{i}=t_{i}{ }_{i}+i t^{\prime \prime}
$$

Accordingly, equation (60) can be written as

$$
\left.\left|\widetilde{g}_{i}\right|=\mid \frac{1-(1-\mu)\left(t^{\prime}{ }_{i}+i t^{\prime \prime}\right.}{i}\right) \mid \leq 1
$$

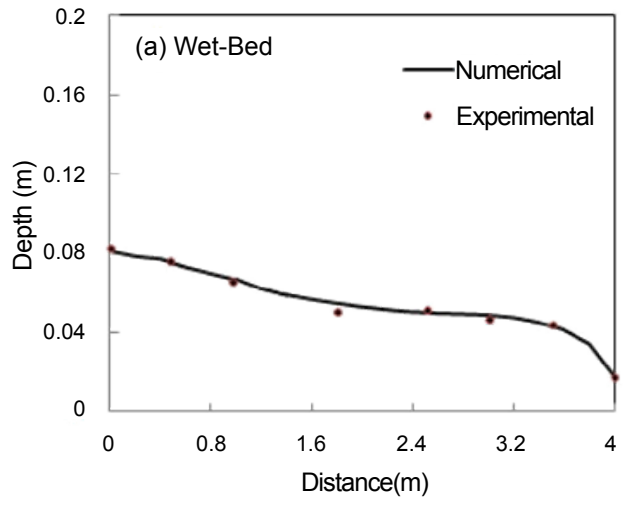

a

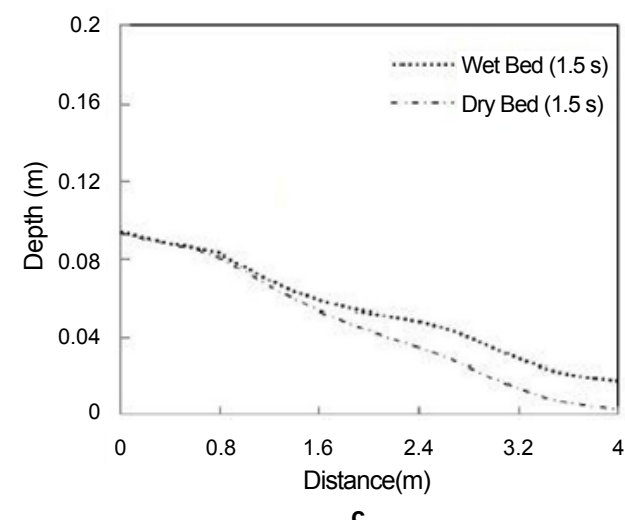

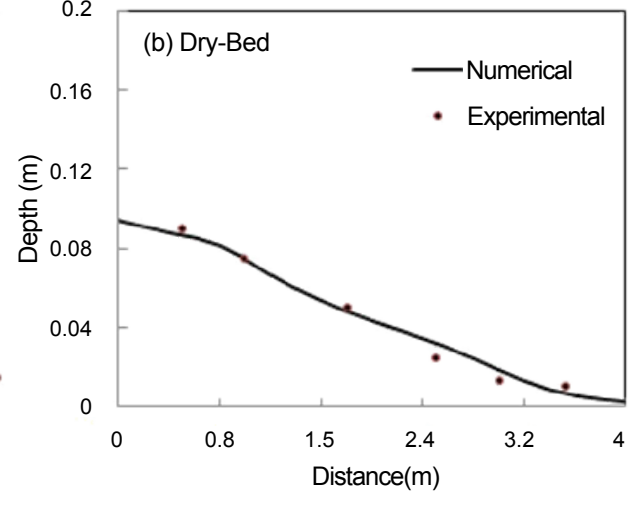

b

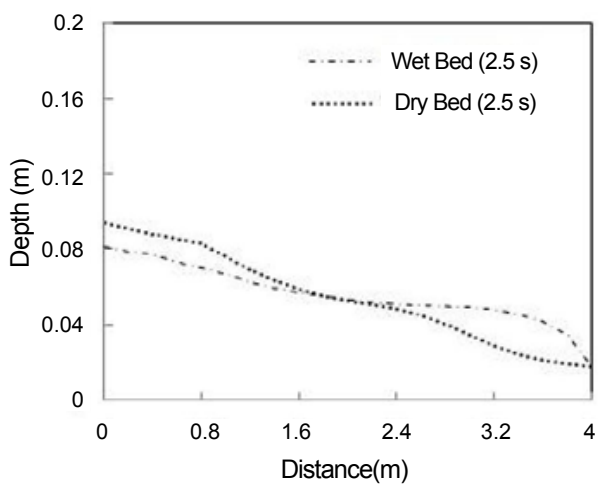

b

Figure 3: a,b: Comparison of depth vs. distance: (a) Wet-Bed at $t=2.5$ (b) Dry-Bed at $t=1.5$. c,d: Comparison of water depth for wet and dry beds for $t=1.5 \mathrm{~s}$ and $2.5 \mathrm{~s}$. 


$$
\begin{aligned}
& \left|1-(1-\mu)\left(t_{i}^{\prime}+i t^{\prime \prime}{ }_{i}\right)\right| \leq\left|1+\mu\left(t_{i}^{\prime}+i t_{i}{ }_{i}\right)\right| \\
& \left(1-(1-\mu) t_{i}^{\prime}\right)^{2}+(1+\mu)^{2} t_{i}^{\prime \prime 2} \leq\left(1+(\mu) t_{i}^{\prime}\right)^{2}+(\mu)^{2} t_{i}^{\prime \prime 2} \\
& 1+t_{i}^{\prime 2}+(\mu)^{2} t_{i}^{\prime 2}-2(\mu) t_{i}^{\prime 2}-2(1-\mu) t_{i}^{\prime \prime 2} \\
& +(\mu)^{2} t_{i}^{\prime \prime 2}-2(\mu) t_{i}^{\prime \prime 2} \leq 1+(\mu)^{2} t_{i}^{\prime 2}+2(\mu) t_{i}^{\prime}+(\mu)^{2} t^{\prime \prime 2}{ }_{i} \\
& (1-2 \mu)\left(t_{i}^{\prime 2}+t_{i}^{\prime \prime 2}\right)-2 t_{i}^{\prime} \leq 0
\end{aligned}
$$

This indicates that the method is stable provided the above condition is satisfied.

\section{Conservative form}

For conservative form the matrix $\mathrm{T}$ can be obtained by substituting equations (37)-(39) in equation (59)

$$
T_{i j}=\frac{3}{(1+\cos \theta+\cos \gamma)}\left(\frac{1}{\Delta y} i \sin \gamma B_{i j}+\frac{1}{\Delta x} i \sin \theta A_{i j}\right)
$$

Since in equation (67) real part is zero (Appendix-1), $t_{i}=i t^{\prime \prime}{ }^{*}$ Accordingly, equation (66) becomes

$$
(1-2 \mu) t_{i}{ }_{i}^{2} \leq 0=>\geq 1 / 2
$$

It means that the method is unconditionally stable for $\mu \geq 1 / 2$.

\section{Numerical Experiment and Model Validation}

To demonstrate the validity and effectiveness of the proposed scheme, unsteady 2D dam-break flow problem is considered. The schematic diagram of Dam-break flow in straight walled transition is shown in Figure 1. Numerical computations are made for the following input values which correspond to the experiments conducted by Townson and Al-Salihi [19]. The computational domain comprises of 4 $\mathrm{m}$ long and $0.1 \mathrm{~m}$ wide channel. The length of the channel upstream of the dam is $1.8 \mathrm{~m}$ and the channel downstream is $2.2 \mathrm{~m}$. The initial flow depth on the upstream side of the dam is $0.1 \mathrm{~m}$ for both wet bed and dry bed whereas downstream water depth was assumed to be 0.00001 $\mathrm{m}$ and $0.006 \mathrm{~m}$ respectively. The velocities, $u$ and $v$ at inflow boundary are specified as zero for all times $t$ and the flow depth at this boundary computed by using the following linear extrapolation.

$$
h_{1 j}^{k+1}=2 h_{2 j}^{k+1}-h_{3 j}^{k+1}
$$

The boundary conditions at the downstream end are the same as the initial condition in terms of depth and velocities. The symmetry boundaries are treated at the sidewalls. A higher order compact finite difference implicit scheme is used for numerical simulation on a $21 \times 5$ grid with $\Delta \mathrm{t}=0.01$. The corresponding grid sizes become $\Delta \mathrm{x}=0.2 \mathrm{~m}$ and $\Delta y=0.025 \mathrm{~m}$ along $\mathrm{x}$ and $\mathrm{y}$ directions respectively. To reduce the higher order dissipation due to aliasing error introduced by the fourthorder compact scheme, Wallington filter [32] is applied. This consists of periodic successive application of the following two-point operators where one filtering is performed after eighteen iterations.

$$
\begin{aligned}
& \bar{\varphi}_{i}=4.28 \varphi_{i}-2.16\left(\varphi_{i+1}+\varphi_{i-1}\right)+0.52\left(\varphi_{i+2}+\varphi_{i-2}\right) \\
& \tilde{\varphi}_{i}=0.375 \bar{\varphi}_{i}+0.25\left(\bar{\varphi}_{i i+1}+\bar{\varphi}_{i i-1}\right)+0.0625\left(\bar{\varphi}_{i i+2}+\bar{\varphi}_{i i-2}\right)
\end{aligned}
$$

The numerical wave profiles are compared with the experimental values, $2.5 \mathrm{~s}$ after the instantaneous dam-break for the wet bed conditions whereas for dry bed condition $1.5 \mathrm{~s}$ is considered. Figure 3a shows very good agreement of water surface profiles between numerical and experimental results for wet bed condition. For dry bed condition as shown in figure $3 \mathrm{~b}$, the result shows very good agreement for upstream section and also closer to the downstream boundary. However, in both the cases compact formulation provides much better results as compared to the results of Mahapatra and Bhallamudi [21]. With such coarser discretization, desired accuracy has been achieved in comparison to the traditional schemes.

\section{Results and Discussions}

In this problem, dam breach takes place instantaneously with full breach condition. Due to discontinuous initial conditions, it enforces several restrictions and most of the numerical schemes fail to capture this phenomenon. The algorithm developed here is second order accurate in time and fourth order accurate in space $O\left((\Delta t) p, h^{4}, k^{4}\right)$ , on the nine-point stencil using third order non-centered difference at the wall boundaries. For solving the algebraic systems, we adopt biconjugate gradient stabilized method (BiCGStab) with preconditioning for $21 \times 5$ grid points along $\mathrm{x}$ and $\mathrm{y}$ directions. Interpolation has been performed along y direction while plotting. It is also possible to partition the coefficient matrix to a block tri-diagonal matrix then find Solution by simple block tri-diagonal solvers. We present our results for wet bed and dry bed conditions for downstream of the dam. The actual water depth at $t=0$ is specified as the initial condition in the downstream. The tail water/reservoir ratio for wet bed and dry bed is considered as 0.176 and 0.025 respectively. In the case of dry bed, downstream water level is fixed at $0.0025 \mathrm{~m}$ and below to this level would cause blow up due to not satisfying the continuity equation. Figure $3 \mathrm{c}$ shows the comparison of water depth profiles for wet bed and dry bed conditions at the downstream after $1.5 \mathrm{~s}$ of dam-break. During this time the wave due to dam-break has not reached the upstream boundary. It can be noticed that the water depth profiles for wet bed and dry bed condition remains same till mid location of the upstream section and subsequently water depth profile of wet bed increases gradually towards downstream direction. Figure $3 \mathrm{~d}$ shows the comparison of water depth profiles for wet bed and dry bed conditions for time $t=2.5 \mathrm{~s}$ after the dam-break The wave front clearly indicates the effect of dam break closer to the

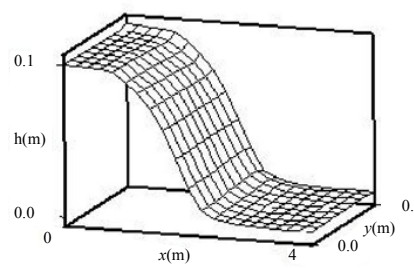

(a) $\operatorname{Dry}-\operatorname{Bed}(0.5 \mathrm{~s})$

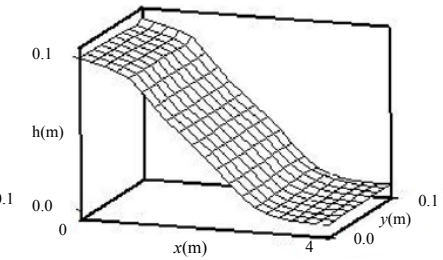

(b) Dry-Bed $(1.0 \mathrm{~s})$

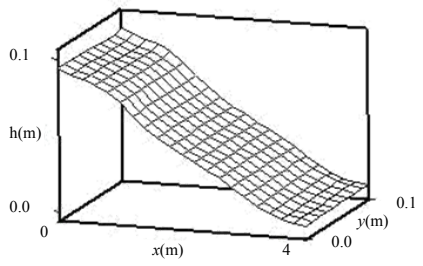

(c) $\operatorname{Dry}-\operatorname{Bed}(0.5 \mathrm{~s})$

Figure 4: a,b: Water surface profiles at $t=0.5 \mathrm{~s}, 1.0 \mathrm{~s}$ after the dam-break for dry bed condition. c: Water surface profiles at $t=1.5 \mathrm{~s}$ after the dam-break for dry-bed condition. 


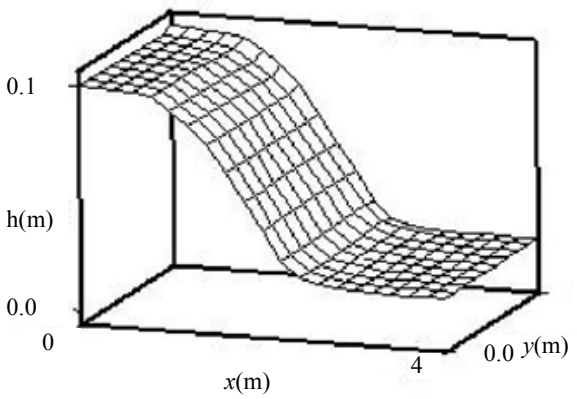

(a) Wet-Bed $(0.5 \mathrm{~s})$

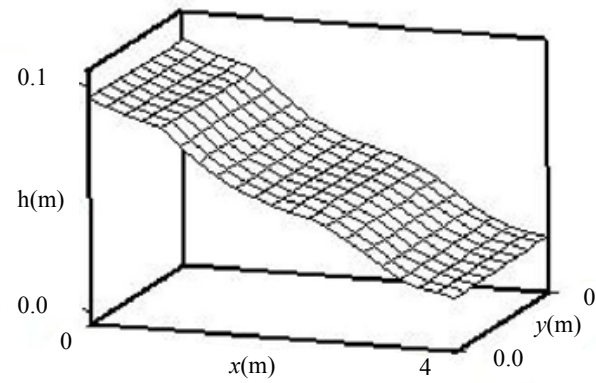

(c) Wet-Bed(1.5 s)

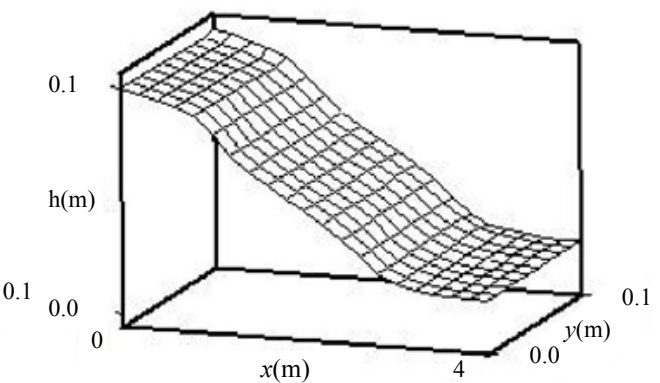

(b) Wet-Bed(1.0 s)

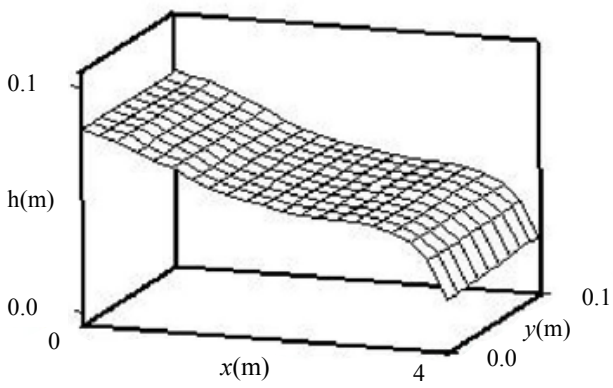

(d) Wet-Bed(2.5 s)

Figure 5: Water surface profiles at $t=0.5,1.0,1.5,2.5$ after the dam-break for wet-bed condition.

downstream boundary for wet bed condition. Figures $4 a-4 c$ show the three-dimensional view of water surface profiles for dry bed taken at time $t=0.5 \mathrm{~s}, 1.0 \mathrm{~s}$ and $1.5 \mathrm{~s}$ respectively. It can be noted that water surface profiles manifest smooth transition even with less number of grid points. At time $t=0.5 \mathrm{~s}$, (Figure $4 \mathrm{a}$ ), the flood wave is yet to reach the downstream region and therefore water level remains at 0.0025 $\mathrm{m}$. Figure $4 \mathrm{~b}$ shows that after $t=1.0 \mathrm{~s}$, the flood wave almost reach downstream region barring couple of grid points close to the boundary. At time $t=1.5 \mathrm{~s}$, the flood wave reaches the downstream completely and drop in water level in the upstream boundary is noticed (Figure 4c). We consider four cases to illustrate the effect of flood wave for wet bed conditions for time $t=0.5 \mathrm{~s}, 1.0 \mathrm{~s}, 1.5 \mathrm{~s}$ and $2.5 \mathrm{~s}$ and the corresponding water surface profiles are shown in Figres 5a-5d respectively. Similar to the dry bed conditions, the flood wave does not reach the downstream completely at time $t=0.5 \mathrm{~s}$ and $1.0 \mathrm{~s}$ as shown in Figures $5 \mathrm{a}$ and $5 \mathrm{~b}$ respectively. Nevertheless, corresponding rise in the downstream water levels is due to the imposition of higher water level during initial condition (wet bed). Figure $5 \mathrm{c}$ and $5 \mathrm{~d}$ show that the wave due to dambreak has reached upstream end with significant decrease in flow depth and the movement of wave front through numerical simulation.

\section{Conclusions}

In this paper we have developed two types of fourth order compact schemes for steady as well as unsteady cases using non-conservation and conservative forms for two-dimensional shallow water equations. This fourth order compact scheme is applied for dam-break problem and the numerical results are compared well with the experimental results. Due to the implicit nature and less stringent stability criteria, the present model proved to be more versatile even with coarser grids and can provide a useful framework for spatial discretization. It is also possible to partition the coefficient matrix to block tridiagonal matrix and then obtain solution by using the simple tridiagonal solver. The present model could be a good alternative to the existing explicit MacCormack [27] or implicit Beam and Warming [33] methods for solving hyperbolic systems in conservative law form, particularly for solving the shallow water equation (SWE).

\section{References}

1. Navon IM, Riphagen HA (1979) An Implicit Compact Fourth-Order Algorithm for Solving the Shallow-Water Equations in Conservation-Law Form. Mon Wea Rev 107: 1107-1127.

2. Abarbanel S, Kumar A (1988) Compact high-order schemes for the Euler equations. Journal of Scientific Computing 3: 275-288.

3. Gupta MM (1991) High accuracy solutions of incompressible Navier-Stokes equations. J Comput Phys 93: 343-359.

4. Lele SK(1992) Compact finite difference schemes with spectral-like resolution. J Comput Phys 103: 16-42.

5. Spotz WF, Carey GF (1995) Higher-order compact scheme for steady streamfunction vorticity equations. Int J Numer Meth Eng 38: 3497-3512.

6. Li M, Tang T (2001) A Compact Fourth-Order Finite Difference Scheme for Unsteady Viscous Incompressible Flows. Journal of Scientific Computing 16: $29-45$.

7. Kalita JC, Dass AK, Dalal DC (2004) A transformation-free HOC scheme for steady convection-diffusion on non-uniform grids. Int J Numer Meth FI 44: 33-53.

8. Pandit SK, Kalita JC, Dalal DC (2007) A transient higher order compact schemes for incompressible viscous flows on geometries beyond rectangular. J Comput Phys 225: 1100-1124.

9. Kalita JC, Dass AK, Nidhi N (2008) An efficient transient Navier-Stokes solve on compact nonuniform space grids. J Comput Appl Math 214: 148-162.

10. Shah A, Li Y, Khan A (2010) Upwind compact finite difference scheme for timeaccurate solution of the incompressible Navier-Stokes equations. Appl Math Comput 215: 3201-3213. 
Citation: Bagheri J, Das SK (2013) Modeling of Shallow-Water Equations by Using Implicit Higher-Order Compact Scheme with Application to Dam-Break Problem. J Appl Computat Math 2: 132. doi:10.4172/2168-9679.1000132

Page 9 of 9

11. Li M, Tang T, Fornberg B (1995) A compact fourth-order finite difference scheme for the steady incompressible Navier-Stokes equations. Int J Numer Meth FI 20: 1137-1151.

12. Dressler RF (1954) Comparison of Theories and Experiments for the Hydraulic Dam-Break Wave. International Association of Scientific Hydrology 38: 319328.

13. Yevjevich V, Barnes AH (1970) Flood Routing Through Storm Drains - Part II, Physical Facilities and Experiments. Hydrology papers, No. 44, Colorado State University, Colorado, USA.

14. Lauber G, Hager WH (1998) Experiments to dambreak wave: Horizontal channel. J Hydraul Res 36: 291-307.

15. Spinewine B, Zech Y (2007) Small-scale laboratory dam-break waves on movable beds. J Hydraul Res 45: 73-86.

16. Xanthopoulos Th, Koutitas Ch (1976) Numerical Simulation of a TwoDimensional Flood Wave Propagation due to Dam Failure. J Hydraul Res 14: 321-331.

17. Katopodes N, Strelkoff T (1978) Computing two-dimensional dam-break flood waves. J Hydraul Div ASCE 104: 1269-1288.

18. Fread DL, Lewis JM (1988) FLDWAV: A Generalized Flood Routing Model. Proceedings of National Conference on Hydraulic Engineering, Colorado Springs, Colorado, 666-673.

19. Townson JM, Al-Salihi AH (1989) Models of Dam-Break Flow in R-T Space. J Hydraul Eng 115: 561-575.

20. Bellos V, Hrissanthou V (2011) Numerical Simulation of Dam-Break Flood Wave. European Water 33: 45-53.

21. Mohapatra PK, Bhallamudi SM (1996) Computation of a dam-break flood wave in channel transitions. Adv Water Resour 19: 181-187.
22. Zoppou C, Roberts S (2000) Numerical solution of the two-dimensional unsteady dam-break. Appl Math Model 24: 457-475.

23. Arico C, Nasello C, Tucciarelli T (2007) A marching in space and time (MAST) solver of the shallow water equations, Part II: The 2-D model. Adv Water Resour 30: 1253-1271.

24. Chaudhry HM (2010) Open-Channel Flow. (2ndedn), Springer-Verlag, New York, USA.

25. Biscarini C, Di Francesco S, Manciola P (2010) CFD modeling approach for dam-break flow studies. Hydrol Earth Syst Sci 14: 705-718.

26. Bellos CV, Soulis JV, Sakkas JG (1991) Computation of two-dimensional dambreak induced flows. Adv Water Resour 14: 31-41.

27. Mac Cormack RW (1969) The effect of viscosity in hypervelocity impact cratering. AIAA paper, 354.

28. Lax P, Wendroff B (1960) Systems of conservation laws. Commun Pur App Math 13: 217-237.

29. Adam Y (1977) Highly accurate compact implicit methods and boundary conditions. J Comput Phys 24: 10-22.

30. Gustafsson B (1975) The Convergence Rate for Difference Approximations to Mixed Initial Boundary Value Problems. Math Comput 29: 396-406.

31. Henrici P (1974) Applied and computational complex analysis, Wiley.

32. Wallington CE (1962) The use of smoothing or filtering operators in numerical forecasting. Q J Roy Meteor Soc 88: 437-484.

33. Beam RM, Warming RF (1978) An Implicit Factored Scheme for the Compressible Navier-Stokes Equations. AIAA Journal 16: 393-401. 